\title{
Los propósitos de la formación profesional: acerca de las competencias
}

\author{
Miguel Ángel GIRADO QUISPE
}

\section{RESUMEN}

Pareciera que hasta el momento, a pesar de las críticas realizadas a la elaboración de un currículo por competencias, no está bien precisado el papel de los objetivos educativos. De tal manera, al no ser precisados estos, es posible no tener una orientación exacta de los propósitos de la educación, principalmente, la profesional.

Asimismo, en la estructura de los sílabos universitarios mecánicamente se ha ido asimilando las competencias de la misma manera con la que se pretendió aplicar en la educación básica, sin ninguna, reflexión y sin ningún fundamento que amerite su viabilidad y dejando, muchas de las veces, fuera de la lógica de la construcción del sílabo a los objetivos. Es decir, no se menciona en ninguna parte del sílabo los objetivos educativos. Quedando, definitivamente, sin una orientación precisa, sin derrotero, los contenidos a enseñar.

Ahora bien, según Delors, Alonso y Peñaloza las competencias se patentizan en el hacer. Pero en una formación profesional, si bien es cierto el hacer cumple un papel relevante, es insuficiente. Ya que, un egresado debe, también conocer los fundamentos teóricos de la profesión elegida. Y a esta situación se debe problematizar los valores, considerando que a la edad que ingresan los alumnos a las universidades se insertan con determinados valores que guían su comportamiento. Además en la universidad no sólo se debe formar para el trabajo; sino, también, que sean capaces de involucrarse en los problemas latentes de nuestra sociedad para darle solución.

Ahora bien, todo objetivo tiene su referente, pues está dirigido hacia algo. En el caso de la educación, en general, está determinada teleológicamente, pues, la acción humana es intencional. En el caso educativo, la / formación profesional es un acto humano. Por tanto, exige objetivos, a menos que se sostenga que el accionar humano es un proceso instintivo o automático. Aspecto que no tendría ningún sustento para asumirlo como verdadero. Entonces, un 
sílabo debe tener como contenido orientador, como derrotero los propósitos de formación, es decir, los objetivos; respetando la estructura técnica de su elaboración. En la actualidad existe una gran preocupación de los que dirigen las universidades. Pues surge la necesidad de lograr ser acreditados, a nivel nacional o internacional; entonces se apertura la posibilidad de revisar las orientaciones metodológicas de estos organismos acreditadores; de tal manera generar innovaciones curriculares.

En tanto a la estructura o la lógica del sílabo, hay mucho por realizar, no obstante la importancia del mismo, ya que es ahí donde se patentiza el perfil. Es decir, el sílabo es el documento que va orientar la formación profesional. Pues, en la relación espacio temporal se tiene el primer contacto con aquellos que van a ser formados y que van a ser teleológicamente determinados por el perfil.

\title{
Descriptores: Competencias y objetivos
}

\begin{abstract}
It seems that so far, despite the criticisms made in the development of a competency-based curriculum is not well clarified the role of educational objectives. Thus, not being stated objectives, it may not have a precise orientation of the aims of education, mainly vocational education.
\end{abstract}

Likewise, in the structure of university syllabi mechanically has assimilated the powers of the same way they were intended to apply to basic education. Without any, reflection and without any basis to warrant its viability. Leaving, many times without building the logic of the objectives of the syllabus. That is, is not mentioned anywhere in the syllabus of educational objectives. Being, definitely, without a clear focus, no road map to teach content.

However, according to Delors, Alonso, Peñaloza skills are manifested in the doing. But in professional training, although making plays an important role, is insufficient. Since, a gradúate must also understand the theoreticai underpinnings of their chosen profession. And this situation is to problematize the valúes, considering that at age students entering the universities already have a scheme of values.

Now, every lens has as its referent is directed towards something. In the case of education, in general, is determined teleologically. Well, human action is intentional. In the case of education, vocational training is a human act. Therefore, requires objectives, unless it is held that human action is instinctive or automatic process. Appearance would not have any basis to assume it as true. Then, a syllabus should be as content advisor, as sailing training purposes, the objectives, respecting the technical structure of its production. There is 
now a major concern of those who run universities. Then comes the need to be accredited, national or international level, then opening the possibility of reviewing the methodological orientations of these accreditation bodies, such curricular innovations genérate.

In both structure and logic of the syllabus, there is much to be done, despite the importance of it, because that's where the profile, are manifested. That is, the syllabus is the document that will guide the training. Well, in the temporary space ratio is the first contact with those who will be trained and that will be teleologically determined by the profile.

\section{Keywords: Competencies, objectives.}

\section{INTRODUCCIÓN}

\section{Aproximación al estado del arte de la competencia}

El sentido etimológico: Peñaloza (2003) menciona que se origina en el latín "competentia, competens, respectivamente. A su vez estos términos se formaron de fos vocablos cum= 'con' y peto (infinitivo: petere = 'ir' y también atacar). Cum + petare quiere decir: (a) 'ir con', 'ir a la par con otro', 'ir a la par con algo', 'ser adecuado a algo'; y también (b) luchar con otro', 'atacar a otro'. En castellano en el primer sentido se expresa con el verbo 'competir' y el segundo con el verbo 'competir'. Nuestro sustantivo 'competencia' se relaciona ya con el primer sentido, ya con el segundo. En cambio, nuestro adjetivo 'competente' se relaciona sólo con el primero (para el segundo decimos competidor). En efecto, en latín competens quería decir 'adecuado para algo', 'proporcionado a algo', 'idóneo para algo', 'algo que va a la par de algo', 'que es apropiado para algo', de donde nace una tercera noción, de carácter jurídico (y también burocrático): el juez que es apropiado para conocer de un asunto es el juez competente, tiene la competencia necesaria para intervenir" (p. 48).

La competencia en los griegos: Peñaloza (2003) nos dice que: "Platón al hablar de la techné (profesiones, artesanías y oficios), realiza entre los conocimientos necesarios (la 'episteme') y las acciones reales respectivas ('epiteudema'). Cada 'techné' es la simbiosis de las dos cosas y únicamente con las dos cosas un 'technites' (profesional, artesano u obrero) es competente. Si esa es la 'techné' conlleva el aprendizaje de los conocimientos pertinentes y el aprendizaje de las acciones reales propias del trabajo correspondiente" (p. 52).

\section{La competencia en el informe a la UNESCO de la Comisión Internacional} sobre la educación para el Siglo XXI presidida por Jacques Delors (1996): "Cada vez con más frecuencia, los empleadores ya no exigen una calificación determinada, que consideran demasiado unida todavía a la idea de pericia material, y piden, en cambio, un conjunto de competencias específicas a cada 
persona, que combina la calificación propiamente dicha, adquirida mediante la formación técnica y profesional, el comportamiento social, la aptitud para trabajar en equipo, la capacidad de iniciativa y la de asumir riesgos.

Si a estas nuevas exigencias añadimos la de un empeño personal del trabajador, considerado como agente del cambio, resulta claro que ciertas cualidades muy subjetivas, innatas o adquiridas -que los empresarios denominan a menudo «saber ser»- se combinan con los conocimientos teóricos y prácticos para componer las competencias solicitadas; esta situación ilustra de manera elocuente, cómo ha destacado la Comisión, el vínculo que la educación debe mantener entre los diversos aspectos del aprendizaje. Entre esas cualidades, cobra cada vez mayor importancia la capacidad de comunicarse y de trabajar con los demás, de afrontar y solucionar conflictos. El desarrollo de las actividades de servicios tiende a acentuar esta tendencia" (pp. 49-50).

La competencia según Programa Especial Mejoramiento de' la Calidad de la Educación Peruana (MECEP) y Ministerio de Educación del Perú (MINEDU): La competencia "Es la capacidad de la persona para construir y utilizar conocimientos, resolver problemas, tomar iniciativas y desempeñarse con eficacia, eficiencia y satisfacción frente a cualquier aspecto de su realidad.

Cada competencia viene a ser un aprendizaje complejo que integra las siguientes dimensiones: Saber: aprendizajes teóricos que servirán de base para la elaboración de conceptos cada vez más complejos como producto del proceso constructivo del aprendizaje. Éste es el llamado componente conceptual de la competencia.

Saber hacer: Dominio de procesos y tecnologías -como participar en grupo, investigar, realizar experimentos, resolver problemas, operar, comunicar en forma oral o escrita-; interiorización de formas de llegar al conocimiento. Se trata del componente procedimental.

Ser: Desarrollo de actitudes y valores más directamente vinculados al comportamiento social y al desarrollo ético. Se refiere al respeto por sí mismo, a la autoestima y a la capacidad de valorar a las personas, la naturaleza, hechos y situaciones vinculadas a la vida. Es el componente actitudinal de la competencia.

Estas dimensiones son complementarias, aunque con frecuencia alguna de ellas articula el aprendizaje" (p. 17).

Las competencias según Martha Alles: Alles (2000) asume la definición de competencia de Ernst \& Young, nos va a decir que la competencia que garantiza el éxito son: 
1. "Los conocimiento: que derivan de la aplicación de una técnica específica.

2. Las habilidades: que normalmente se adquieren mediante entrenamiento y experiencia.

3. Las cualidades: algunas están relacionadas con rasgos o características personales y son más difíciles de obtener y modificar en corto plazo" (pp. 56-57).

Asimismo, nuestra autora siguiendo a Spencer \& Spencer nos aclara que la competencia es: "Característica subyacente" porque "es una parte profunda de la personalidad y puede predecir el comportamiento en una amplia variedad de situaciones y desafíos laborales". También es "estándar de efectividad" que "la competencia realmente predice quién hace algo bien o pobremente, medido sobre un criterio general o estándar."

Y "causalmente relacionada", menciona por qué, "la competencia origina o anticipa el comportamiento o desempeño” (p. 59).

Las competencias según Tobón (2008): Define "las competencias son procesos complejos que las personas ponen en acción-actuación-creación, para resolver problemas y realizar actividades (de la vida cotidiana y del contexto laboralprofesional), aportando a la construcción y transformación de la realidad, para lo cual integran el saber (automotivación, iniciativa y trabajo colaborativo con otros), el saber conocer (observa, explicar, comprender y analizar) y el saber hacer (desempeño basado en procedimientos y estrategias), teniendo en cuenta los requerimientos específicos del entorno, las necesidades personales y los procesos de incertidumbre, con autonomía intelectual, conciencia crítica, creatividad y espíritu de reto, asumiendo las consecuencias de los actos y buscando el bienestar humano. Las competencia en tal perspectiva están constituidas por procesos subyacentes (cognitivo afectivos) así como procesos públicos y demostrables, en tanto implican elaborar algo de sí para los demás con rigurosidad" (p. 49).

Una definición en el contexto de la sociedad del conocimiento: Román (2005) considera la competencia como "las capacidades -destrezas y valoresactitudes, contenidos sintéticos y sistémicos y métodos o habilidades, donde el contenido y el método son medios para desarrollar capacidades y valores, tanto profesionales como educativos (para la vida). Nos interesa no sólo la manifestación observable de una competencia como conducta (conductismo y sociedad industrial) sino lo subyacente en la misma en forma de capacidades y valores. De este modo posibilitaremos la transición y redefinición del concepto de competencia y perfil profesional en el marco de la sociedad del conocimiento y la Refundación de la Escuela y la Universidad, en una nueva sociedad (cuyo capital es el conocimiento como producto del aprendizaje permanente)" (p. 12) 
La definición según Zabalza (2007): Menciona las virtualidades de las competencias como:

1. Las competencias orientan la enseñanza hacia la acción. Podríamos decir que, en cierta manera, cierran el círculo del aprendizaje incluyendo en él, como elemento sustantivo el de la acción. Rompen por tanto, con una enseñanza demasiado centrada, en ocasiones, en los saberes (en el saber por el saber). Nuestros alumnos aprenden (asimilan mentalmente), con frecuencia, grandes cantidades de informaciones con el único objetivo de hacerse con ellas, de dominarlas en tanto que unidades informativas de contenido cultural o científico. Pero no tienen la oportunidad de aplicarlas, de usarlas en procesos que lleven a la consecución de algún resultado práctico (salvo el reproducir ese conocimiento en un examen cuyo resultado será la calificación recibida).

Cuando hablamos de competencias estamos hablando de un saber que me permite hacer algo nuevo (hacer algo que no sabía hacer o hacerlo en mejores condiciones de las que las hacía). De esta manera, los saberes convencionales se convierten, como señala Perrenoud (2004), en "recursos para la acción". La acción, la realización de algún tipo de proceso práctico resulta básico para visualizar la efectiva adquisición de la competencia.

2. Las competencias integran diversos componentes del aprendizaje. No basta con el mero saber o la simple erudición para desenvolver el proceso, completo de la actividad recogido en la competencia. Saber las reglas de un juego no me hace competente en dicho juego: preciso poseer las habilidades precisas para desarrollarlo, controlar el proceso o secuencia de operaciones a llevar a cabo y contar con las actitudes que me permitirán actuar adecuadamente en la situación. Por eso el trabajo en base a competencias exige tanto conocimientos como práctica; exige un ejercicio suficiente de la acción y la capacidad de estar y sentirnos de una forma conveniente a las condiciones del desarrollo de dicha acción.

3. Las competencias implican procesos cognitivos distintos del mero aprendizaje asimilativo: la competencia moviliza e integra los distintos componentes del saber.

Incluso en el caso de que poseamos los recursos que integran una competencia (conocimientos, habilidades, procesos operativos y actitudes), a veces aprendidos de forma aislada o consecutiva, precisamos de situaciones en las que podamos integrarlos en una situación realista. De ahí la importancia de las simulaciones, de las prácticas de laboratorio o de las prácticas sobre el terreno (el practicum). Sólo en esos contextos más próximos a la realidad profesional estamos en condiciones de poder movilizar el conjunto de nuestros recursos vinculados a la competencia. 
"Las competencias se adquieren típicamente en un proceso de aprender haciendo' -learning by doing-en situaciones reales de trabajo, durante las prácticas externas-internship-, o en una situación de aprendizaje basado en simulaciones" (Roe, 2003)

Por eso decimos que la competencia es "saber actuar adecuadamente en situaciones concretas". Supone superar el "saber cómo habría que actuar" (planteamiento muy habitual en la formación universitaria), al "ser capaz de actuar de hecho" (lo que implicaría la oportunidad - exigencia de actuar como parte del proceso de formación).

4. Las competencias implican actuaciones en situaciones complejas. Los niveles de actuaciones pueden ser muy diversos por lo que también lo son las capacidades necesarias para llevar a cabo tales actividades. De hecho esta es una distinción básica para clarificar parte del debate en torno a las competencias.

Las situaciones simples, puramente actuárteles, pueden ser resueltas a través de meras habilidades operatorias o skills. Los sujetos pueden aprender a hacer cosas por la simple asimilación de la cadena operativa que lleva a la obtención del producto o resultado apetecido. Pero estaríamos ante una habilidad no ante una competencia que implica necesariamente una integración del saber y el hacer ( $\sin$ que se reduzca ese saber a cómo hacer la cosa sino a conocimientos más amplios de la situación)"1.

Las competencias profesionales según CONEAU (2009) "La Competencia Profesional se identifica en las normas a través de la definición de elementos de competencia (logros laborales que un trabajador es capaz de conseguir), criterios de desempeño (definiciones acerca de la calidad), el campo de aplicación y los conocimientos requeridos" (p. 23).

\section{Las competencias según la Organización Internacional del Trabajo} (OIT) es definido como la "Capacidad de articular y movilizar condiciones intelectuales y emocionales en términos de conocimientos, habilidades, actitudes y prácticas, necesarias para el desempeño de una determinada función o actividad, de manera eficiente, eficaz y creativa, conforme a la naturaleza del trabajo. Capacidad productiva de un individuo que se define y mide en términos de desempeño real y demostrando en determinado contexto de trabajo y que no resulta solo de la instrucción, sino que, de la experiencia en situaciones concretas de ejercicio ocupacional."

Vicente Santiváñez (2007) define las competencias como "macrohabilidades, desempeños o comportamientos que involucran contenidos, los cuales son de

1 Recuperado el día 14 de setiembre de 2011; de: http://www.upd.edu.mx/varios/simpdidac2007/Zabalza.pdf 
tres tipos de saberes o aprendizajes, tales como: cognitivos o conceptuales (saber), procedimentales (saber hacer), afectivos o actitudinales (saber ser)" (p. 109).

\section{El sentido de los objetivos educativos}

La Real Academia Española define objetivo como: "Punto o zona que se pretende alcanzar u ocupar como resultado de una operación militar." Esta definición permitirá determinar el término objetivo en el sentido que consideraremos en esta investigación, es decir, es el comportamiento que se pretende alcanzar como resultado de una operación educativa. Vendría a ser el resultado del aprendizaje. Esto nos permite determinar que el objetivo es el resultado que se espera obtener después del proceso enseñanza-aprendizaje. Gimeno (1997) evocando a Tyler de cómo se concretiza los objetivos presenta el siguiente cuadro (P-29):

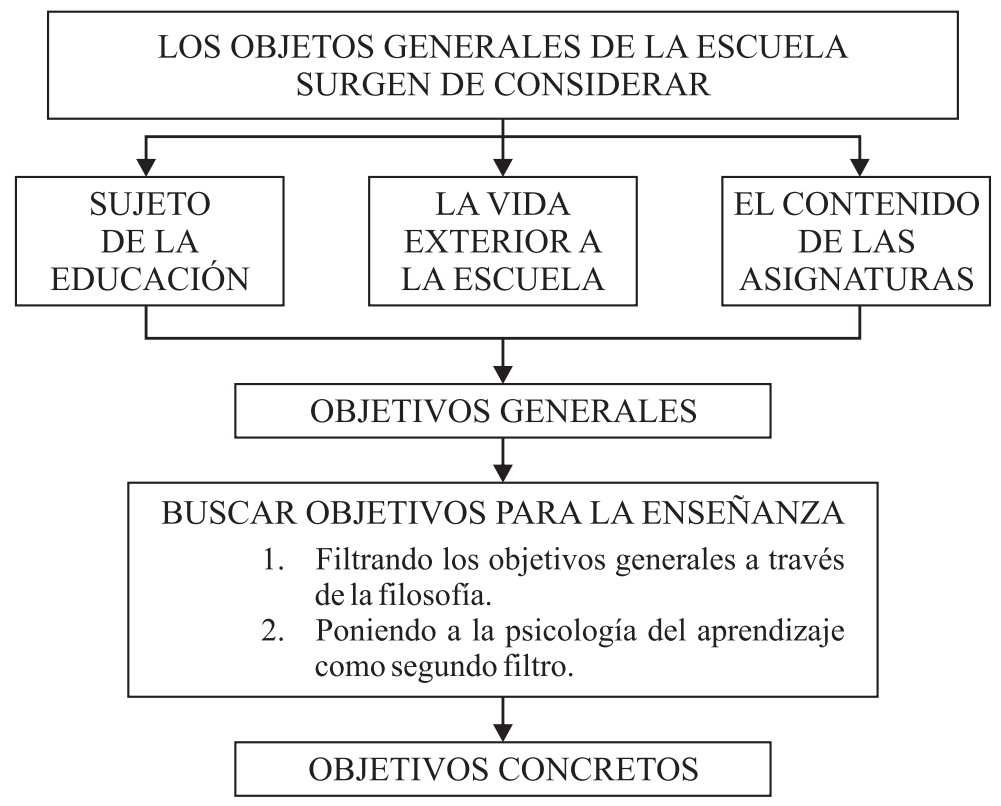

Matheny y Rahmlow (1985) consideran que "Un objetivo es una meta o un propósito. Siempre hablamos de metas y propósitos. Y la gente siempre se explica mutuamente cosas, de acuerdo con metas y propósitos.” (p. 10). 


\section{Antecedentes}

Uno de los antecedentes de los objetivos, según Gimeno (1997) es "El enfoque utilitarista al servicio de la eficiencia social y de la reproducción social configura un marco para la teoría y desarrollo de la enseñanza, de suerte que quedan indicados no sólo los contenidos de la enseñanza y los objetivos de la misma(las actividades humanas), sino también una metodología para la búsqueda de esos objetivos -el análisis de tareas- y una forma de planificar la enseñanza -tecnología- tomada de la industria.” (p. 19).

Respecto al sentido de la universidad Salazar (1967) menciona que el sentido de la universidad "es el ejercicio y primado del pensamiento racional" (p. 8).

Es absurdo oponerse o divorciarse de la razón crítica, pues, "la comunicación de los pensamientos que se exponen al análisis y a la prueba, que pueden ser sometidos a discusión, confirmados o rectificados" es esencial en la universidad. Ahora bien, frente a la pretensión de elaborar un curriculum por competencias Peñaloza (2003) nos dice que "la pretensión de que un curriculum sea íntegramente por competencias resulta un despropósito. Para empezar, hay un objetivo en todo curriculum, objetivo que ostenta carácter cognoscitivo. Para la educación de los discentes en todos los niveles tenemos que brindarles conocimientos relativos al mundo y también los vinculados al trabajo y sobre ello, o con ello, fomentar su espíritu inquisitivo y la formación de su intelecto, en su dimensión razonadora. Pero el conocer no es una competencia. Es una capacidad interna...

Otro objetivo urgente es que los alumnos abran en su mente horizontes que les permitan entender, aparte de su profesión, los grandes problemas de la humanidad, de su época y de su propio país,... y esto tampoco es una competencia." (p. 121).

\section{Justificación}

El nivel de pobreza en el Perú va hasta el 85.7\% en el 2007 en el Departamento de Huancavelica, la población analfabeta en el Perú es un 7\% al 2010, la malnutrición expresada en peso haciende a un 5\% y la talla en un 30\% al 2008 . De acuerdo al reporte del Instituto Nacional de Estadística e Informática (INEI), en el trimestre móvil diciembre - enero febrero 2011 la tasa de Desempleo en Lima Metropolitana creció más de $22.3 \%$ respecto al trimestre anterior (noviembre, diciembre 2010 -enero), pasando de $360 \mathrm{mil} 600$ (7,7\% de la Población Económicamente Activa -PEA) a 437 mil 100 (9,1\% de la PEA). Del total de desempleados, el $42,4 \%$ son hombres y el $57,6 \%$ son mujeres. ${ }^{2}$

2 Recuperado el día 21 de diciembre de 2012; de: http://www.diariolaprimeraperu. com/online/economia/des empleo-crecio-22-en-la-capitaI_81932.html 
¿Quiénes serán los hombres que tendrán el reto se solucionarlo? ¿De dónde saldrán esos hombres?

Asimismo, si se revisan los sílabos de algunas universidades se observa que carecen de objetivos, Entonces esta realidad presentada exige que se revisen los fundamentos de los contenidos de aprendizaje universitario y su elaboración técnica. De tal manera poder demostrar estas debilidades que de alguna manera no permitiría resolver los problemas nacionales, ya que es en la universidad donde se deben formar los cuadros que tendrán como responsabilidad regir, de alguna manera, los destinos de nuestra patria. Por tanto demostrar que es insuficiente una formación para el trabajo en la universidad.

\section{Objetivos:}

a) Demostrar que en la elaboración de un sílabo debe estar redactado los objetivos.

b) Demostrar que es insuficiente tener como propósito educativo de la formación universitaria las competencias laborales.

\section{METODOLOGÍA DEL TRABAJO}

En este estudio se ha tenido como base la hermenéutica. En efecto Tobón (2009) menciona a Gadamer, Vattimo, Valencia y Botero quienes consideran que "este enfoque de construcción de conocimiento se basa en un diálogo con el objeto y generan nuevas perspectivas" (p. 10).

Cabe resaltar que, la hermenéutica es un método general por lo que no cuenta de pasos estrictos ni de técnicas específicas. Pero seguiremos la sugerencia de Tobón (2009) para el proceso de interpretación; “(1) Tener en cuenta no sólo el sentida de los textos en su interior sino también la forma cómo se usan y se aplican; (2) formular preguntas clave en torno a los textos para dialogar con ellos y poder hallar respuestas, como ocurre en todo diálogo; (3) relacionar los textos con sus contextos, para comprender su sentido; (4) identificar lo que Gadamer (verdad y método) denomina la carga de la tradición, es decir, los prejuicios que hay en los textos como en el intérprete y que se relacionan con el sentido no develado o encubierto.” (p. 11).

Asimismo, en toda construcción teórica se utiliza el lenguaje en función informativa, es decir, la construcción se realiza con las unidades mínimas de pensamiento; ya que los seres humanos almacenan el conocimiento declarativo en unidades denominadas proposiciones y esto permite construir redes complejas de información ${ }^{3}$. Una característica problemática de toda proposición

3 Gallardo, K.

La Nueva Taxonomía de Marzano y Kendail: una alternativa para enriquecer el trabajo educativo desde su planeación. (2009). Recuperado el día 12 de diciem- 
es la de ser verdadera o falsa. Ahora bien, Popper (1994) menciona que "Kant utiliza la palabra objetivo para indicar que el conocimiento científico ha de ser justificable, independientemente de los caprichos de nadie: una justificación es objetiva si en principio puede ser contrastada y comprendida por cualquier persona." (p. 43).

Popper asume la tesis kantiana mencionando que "la objetividad de los enunciados científicos descansa en el hecho de que puedan contrastarse intersubjetivamente” (p. 43). Ahora bien, el método lógico que utiliza Popper en la falsación es el Modus Tollens. Ya que para él, de lo único que podemos estar seguros acerca de una proposición es de su falsedad; pero no de su verdad.

Asimismo, Hempel (1983) menciona que la prueba de hipótesis en una investigación puede ser directa (p. 20), es decir, se va a realizar un razonamiento sin contrastar empíricamente la hipótesis.

\section{RESULTADOS Y DISCUSIÓN}

Proposiciones probablemente verdaderas:

(1) La competencia es el desempeño idóneo en la realización de tareas.

(2) Las competencias son saberes de ejecución.

(3) La competencia es el aprendizaje de las acciones reales del trabajo.

(4) Las competencias son habilidades que se adquieren mediante entrenamiento.

(5) Las competencias como contenidos conceptuales, procedimentales y actitudinales a la vez.

(6) La competencia como recurso para la acción.

(7) Las competencias son contenidos, sintéticos y sistémicos, y métodos para desarrollar capacidades y valores tanto profesional y educativo.

¿Cuál es el contexto y/o sentido de esas proposiciones?

Primera aproximación contextual; El sentido que toma las competencias tiene como contexto, principalmente, el mundo del trabajo.

Segunda aproximación contextual; la educación debe permitir que el educado pueda insertarse en ese mundo. Aplicación de la falsación:

bre de 2011; de: http://www.eduteka.org/gestorp/recursos/docs/7886-2011 -I0-12756o.pdf 
Modus Tollens: $(\forall \mathrm{x})(\mathrm{Ax} \rightarrow \mathrm{Bx})$

$$
\frac{\sim \mathrm{Ba}}{\therefore \sim \mathrm{Aa}}
$$

Ejemplo:

Esta es una de las competencias en la carrera de Odontología, para certificar a los odontólogos:

\section{Instruir en higiene bucal, hábitos bucales y dieta al individuo según las guías de procedimientos establecidos. ${ }^{4}$}

En esta competencia, el odontólogo (asumimos hipotéticamente a un odontólogo cuyo nombre es Antonio y que es poseedor de esa competencia) asume un papel educativo en la que debe enseñar procedimientos respecto a la higiene, hábitos y dieta en función de una guía. Las proposiciones, presentadas líneas arriba, (1), (2), (3), (4) y (6) superan la prueba del Modus Tollens, es decir, su fórmula lógica es: $(\forall \mathrm{x})(\mathrm{Cx} \rightarrow \mathrm{Tx}) \wedge \mathrm{Ta} . \rightarrow$. Ca. En cambio en (5), su fórmula lógica es: $(\forall \mathrm{x})(\mathrm{Cx} \rightarrow(\mathrm{Fx} \wedge \mathrm{Px} \wedge \mathrm{Ax})) \wedge \sim(\mathrm{Fa} \wedge \mathrm{Pa} \wedge \mathrm{Aa}) . \rightarrow$. $\sim \mathrm{Ca}$ A partir de estas pruebas podemos mencionar lo siguiente:

En la afirmación de la posibilidad de aceptar como verdaderas las proposiciones (1), (2), (3), (4) y (6) no es conciuyente, pues, no podemos estar seguros de su verdad, ya que su forma lógica es la famosa falacia de la afirmación del consecuente. La fórmula lógica de esta falacia es:

$$
\begin{aligned}
& (\forall \mathrm{x})(\mathrm{Cx} \rightarrow \mathrm{Tx}) \\
& \mathrm{Ta} \\
& \therefore \mathrm{Ca}
\end{aligned}
$$

Respecto a (5) si podemos estar seguros de su falsedad, por lo tanto no es aceptable esa proposición, entonces no sería una definición correcta de competencia; algo parecido sucede con (7). Además, el conocer y el hacer tienen propósitos distintos. Peñaloza (2003) mencionando el libro La Educación encierra un Tesoro, de Jacques Delors nos dice: "Conocer es un propósito intelectual y los educandos deben aprender a conocer, es decir, a observar, buscar información, comparar, razonar lógicamente, inferir y formular principios y conclusiones. Hacer es realizar cosas en el mundo real, y constituye un propósito praxeológico en que el saber no importa o en que el saber se encuentra deliberada o indeliberadamente ínsito en las acciones ejecutadas.” (pp. 205-206).

Asimismo, Peñaloza, apelando a Platón, nos dice que en la Techné es crucial el epiteudema (la acción o acciones), porque “el saber en sí (episteme) nada tiene

4 Recuperado el día 22 de diciembre del 2011; de: http:/www. scribd.com/fullscreen/6980048 ] ?access_key= key-2abrOzreOujtflyapi85 
que ver con su aplicación a la realidad” (p. 206) Respecto a la actitud, Peñaloza, menciona que "No es un conocimiento: la actitud es una posición frente al entorno, un posicionamiento profundo de la persona ante la realidad; pueden las actitudes (...) contener elementos cognitivos, pero las actitudes no son conocimientos y mal se hace en presentar a las actitudes como conocimiento o saberes. (Además) determinadas actitudes están presentes en las competencias, por ejemplo, el empeño en hacer las cosas bien, el compromiso con la tarea emprendida y el hacerla con seriedad y con el firme propósito de concluirla a cabalidad, pero asimismo está presente en todo lo demás, más allá de las competencias." (p. 225).

Otro autor que confirma la falsación de las competencias como contenidos conceptuales, procedimentales y actitudinales es Carlos Barriga (2004) y confirma la competencia como epiteudema (hacer).

Ahora bien, la formación en competencias laborales, si bien es cierto, aunque importante es, sin embargo, insuficiente, en tanto que es profesionalizante. Pues, la universidad debe asumir el reto que de sus aulas deben salir los que van a dar soluciones concretas a los grandes problemas de nuestro país. Por tanto, se tiene que generar La Formación y Fortalecimiento de la Identidad, Desarrollar la Investigación Científica, Tecnológica e Innovación, Desarrollar Espacios de Razón Crítica, Conocer la Realidad Nacional y Mundial y Educar Hombres Constructores de un Nuevo Tipo de Sociedad en la que el Hombre sea el Fín.

Asimismo, Una redacción de un sílabo en la que no se precise de manera clara los objetivos, no tendría derrotero. Además es insustentable considerar las competencias como una suerte de fusión de tres contenidos: conceptual, procedimental y actitudinal. Pues, por ser teleológico los objetivos, en su redacción se menciona lo que se espera alcanzar del proceso educativo en formación profesional.

SENA (2003) recomienda que en la elaboración de los objetivos "Todas las funciones se enuncian utilizandolaestructuraVERBO+OBJETO+CONDICIÓN. La satisfacción de esta exigencia en la redacción de funciones facilita la descripción estandarizada de resultados laborables evaluables" (p. 28).

"El verbo especifica la acción que realiza el trabajador para obtener el resultado descrito en la función. Cuando se pretende enunciar una función con verbos como conocer, comprender, aprender, apreciar, considerar, saber, entender, estudiar, observar debe hacerse la pregunta ¿Qué resultados laborales puedo obtener cuando conozco, "comprendo, aprendo, aprecio, considero, se, entiendo, estudio, observo? 
La respuesta que se obtenga orienta al equipo y al asesor metodológico hacia el enunciado de la función en términos de resultados evaluables. El objeto es la persona, animal o cosa sobre el cual ocurre la acción del verbo. El conjunto de verbo y objeto especifica una función laboral. La condición define el alcance de la función laboral, expresando un indicador de calidad para la evaluación de la función laboral que se describe con el verbo y el objeto. En la condición se evita el uso de calificativos como: adecuado, correcto, óptimo, completo, preciso, entre otros, porque dificultan una evaluación objetiva e inducen a la especificación de condiciones irreales que exceden el alcance de la función.” (p. 29).

Es necesario precisar que la secuencia para llegar a los objetivos son: Condiciones socioeconómicas, objetivos educativos o de formación profesional, currículo que plasma tales exigencias y propósitos, asignaturas del currículo, y como parte de éstas asignaturas los objeticos de la enseñanza que responden a los propósitos y las demandas de la comunidad. ${ }^{5}$

\section{CONCLUSIONES}

$1^{\circ}$ La competencia en esencia tiene que ver con la realización de tareas o procedimientos.

$2^{\circ}$ La competencia es el desarrollo de capacidades.

$3^{\circ}$ La competencia es insoslayable del mundo del trabajo.

$4^{\circ}$ En la redacción de un sílabo debe precisarse el sentido del proceso educativo.

$5^{\circ}$ El sentido del proceso educativo se determina a través del objetivo de aprendizaje.

$6^{\circ}$ Una formación para el trabajo es insuficiente.

$7^{\circ}$ Las universidades, también, deben formar para dar solución a los graves problemas del país.

$8^{\circ}$ La universidad debe ser un espacio de razón crítica.

\section{AGRADECIMIENTOS}

Al Dr. Carlos Alvarado por haber revisado el borrador del trabajo, y por sus sugerencias.

Al Mg Lucas Palacios por las sugerencias realizadas.

A la Lic. Úrsula Romaní por la revisión de estilo y las recomendaciones vertidas.

5 Propuesto por Lucas Palacios. 


\section{REFERENCIAS}

1. Y. Argudín, Educación competencias: nociones y Trillas, México, 2009.

2. C. Barriga, En tomo al concepto de competencia, En: Educación, revista de la Facultad de Educación de la Universidad nacional Mayor de San Marcos, Lima, Año I, № 1, MAYO 2004.

3. J. De Zubiría, Las competencias argumentativas: la visión desde la educación, Cooperativa Editorial magisterio, Colombia, 2006.

4. M. A. Gallart, Competencias, productividad y crecimiento de! empleo, OIT/Cinterfor, Montevideo, 2008.

5. J. Gimeno, La pedagogía por objetivos: Obsesión por la eficiencia, Ediciones Morata, Madrid, 1997.

6. C. Hempel, Filosofia de la Ciencia Natural, Alianza Editorial, Madrid, 1983.

7. C. Matheny, H. F. Rahmlow, Cómo redactar objetivos de instrucción, Trillas, México, 1985.

8. W. Peñaloza, Los propósitos de la educación: Conocimientos, Capacitación para el trabajo, Actitudes, Vivencias valorativas, Fondo Editorial del Pedagógico San Marcos, Lima, 2003.

9. K. Popper, La lógica de la investigación científica, Tecnos, Madrid, 1994.

10. M. Román, Competencias y perfiles profesionales en la Sociedad del Conocimiento, Ediciones Libro Amigo, Perú, 2005.

11. V. Santiváñez, Diseño curricular, Editor Vicente Santiváñez Limas, Lima, 2007.

12. A. Salazar, Filosofia de la educación universitaria, Facultad de Educación de la UNMSM, Lima, 1967.

13. S. Tobón, Competencias en la educación Superior: políticas hacia la calidad, ECOE Ediciones, Bogotá, 2009.

14. S. Tobón, Formación basada en competencias: Pensamiento complejo, diseño curricular y didáctica, ECOE Ediciones, Bogotá, 2008.

15. SERVICIO NACIONAL DE APRENDIZAJE: SENA (2003). Metodología para la Elaboración de Normas de Competencia Laboral, En Seminario "La experiencia Peruana de Certificación Profesional", CONEAU, Lima, 2011.

E-mail: miurp1@yahoo.es 
